\title{
SS433 as ultraluminous UV source. Comparison with ULXs
}

\section{S. Fabrika* \& O. Sholukhova}

Special Astrophysical Observatory, Nizhnij Arkhyz, Karachaevo-Cherkassia 369167, Russia

E-mail: fabrika@sao.ru

\begin{abstract}
We present optical spectral study of gas filaments located in the nebula W50 surrounding the galactic superaccretor SS 433. We find HeII and [OIII] emission lines in the filaments, the lines have to be produced in the gas photoionization by the SS433. The intrinsic face-on luminosity of the supercritical accretion disk in the far UV region is estimated to be $\sim 10^{40} \mathrm{erg} / \mathrm{s}$. We compare the filament spectra with those of nebulae surrounding the ULXs. We discuss the face-on SS433 spectrum, which is hidden for direct observations, and find it may be similar to the ULX spectra.
\end{abstract}

VII Microquasar Workshop: Microquasars and Beyond

September 1 - 5, 2008

Foca, Izmir, Turkey

*Speaker. 


\section{SS 433 and ultraluminous $X$-ray sources}

SS 433 is the only known persistent superaccretor in the Galaxy, the source of relativistic jets ([14] for review). This is a massive close binary, the compact star is the most probably a black hole $([19,9,20,5])$. SS 433 intrinsic luminosity as believed to be $\sim 10^{40} \mathrm{erg} / \mathrm{s}$, its maximum is in non-observed UV region. It is important that almost all the observed radiation is formed in the supercritical accretion dics, the donor star contributes no more than $5 \%$ of the optical radiation. The huge luminosity suggests the black hole mass to be $\sim 10 \mathrm{M}_{\odot}$. Such a big energy budget of the object is supported by very well measured kinetic luminosity of the jets, $\sim 10^{39} \mathrm{erg} / \mathrm{s}$, both in direct studies of the jets and in studies of the jet powered nebula W $50([11,14])$.

$\mathrm{X}$-ray luminosity of SS 433 is $\sim 10^{35} \mathrm{erg} / \mathrm{s}$, five orders of magnitude less than the bolometric luminosity, the X-rays come from the cooling relativistic jets. At the same time we know that practically all the energy at accretion onto a relativistic star is released in X-rays. This means that the observed radiation of SS 433 was thermalized in a strong wind from the supercritical disc. The wind from a supercritical disc was first predicted by Shakura \& Sunyaev ([37]) and confirmed in radiation-hydrodynamic simulations ([12, 32, 31]). These ideas have led the prediction that SS 433 being observed face-on appeares as an extremely bright X-ray source and we may expect a new type of X-ray sources in external galaxies ([21, 16, 17]).

That is quite probably that the new type of X-ray sources, ULXs (ultraluminous X-ray sources, $[38,29,34])$ are SS 433-like objects observed nearly face-on. Their observed X-ray luminosities are $10^{39}-3 \cdot 10^{41} \mathrm{erg} / \mathrm{s}$ and they are certainly related to massive star population. The disc orientaion in SS 433 is edge-on, with the precessional variations the angle between the disc plane and the line of sight is $10 \pm 20^{\circ}$, so we have not a chance to observe directly the funnel in the supercritical disc. The supercritical disc radiation is not isotropical. If one considers the geometrical beaming (the disc funnel total opening ange $\theta_{f} \sim 50^{\circ}$ [31]) and the property of the locally Eddington radiation of the supercritical discs ([37]), one finds ([18]), that the observed X-ray luminosity of $\sim 10^{41} \mathrm{erg} / \mathrm{s}$ is fairly expected for the face-on SS433 star. However, in spite of such estimates and expectations it is not quite clear, is SS 433 really such luminous object.

SS 433 is 14th magnitude red star, however intrinsically this is very blue, but heavily absorbed object, its derived temperature depends on the dics orientation $([28,8,39,10])$, being about $\sim$ $50000 \mathrm{~K}$ when the disc is the most open to the observer and $\sim 20000 \mathrm{~K}$ when the disc is edgeon. The results were obtained by fitting the observed continuum with an absorbed single black body spectrum. The derived total luminosity of the most open dics was $\sim 10^{40} \mathrm{erg} / \mathrm{s}$. Dolan et al. [10] used the HST (HSP) data in the F277M band together with optical U B V R photometry and cosidered two states of SS 433, "bright" (the most open disc, no eclipses) and "faint" (the disc edge-on). They derived the black body parameters $\mathrm{T}=72000 \pm 20000 \mathrm{~K}, \mathrm{R}=2 \cdot 10^{12} \mathrm{~cm}$ for the bright state and $\mathrm{T}=49000 \pm 20000 \mathrm{~K}, \mathrm{R}=1.5 \cdot 10^{12} \mathrm{~cm}$ (however, the last fit was not significant) for the faint state, and the interstellar extinction $A_{V}=8.2 \pm 0.2 \mathrm{mag}$. They found also an excess in $\mathrm{R}$ band $\left(F_{R}=1.3 \cdot 10^{37} \mathrm{erg} / \mathrm{s}\right)$ not depending on precessional phase, which they attributed to freefree radiation of a gas surrounding the binary. We give here all luminosities for a distance to SS 433 of $5.5 \mathrm{kpc}([4])$.

The high intrinsic luminosity of SS 433 led some groups to a search for SS 433 stars in nearby galaxies $([30,7,15,25])$. The candidates were selected as early $\mathrm{H} \alpha$-emitting stars. SS 433 star in 
M33 would be a bright blue star of $\sim 17$ magnitude $\left(M_{V} \sim-8, M_{b o l} \sim-11\right)$. However, no one SS 433-like star has been discovered yet. The question "is SS 433 really such luminous?" is still important.

The SS 433 optical spectrum is very similar to that of a late WN star ([14]), strong He II emisson supports the high temperature $(\mathrm{T} \sim 50000 \mathrm{~K})$ of the source (the accretion disc wind), however the source geometry is poorly known. In previous studies of SS 433's SED the broad band photometrical data were used and the absorbed single black body source was suggested. The task itself is partly non-correct, because of the far extrapolations of the optical Jeans tail radiation to UV region. Besides that it is not obvious that the supercritical disc wind radiates as a black body (a single black body?).
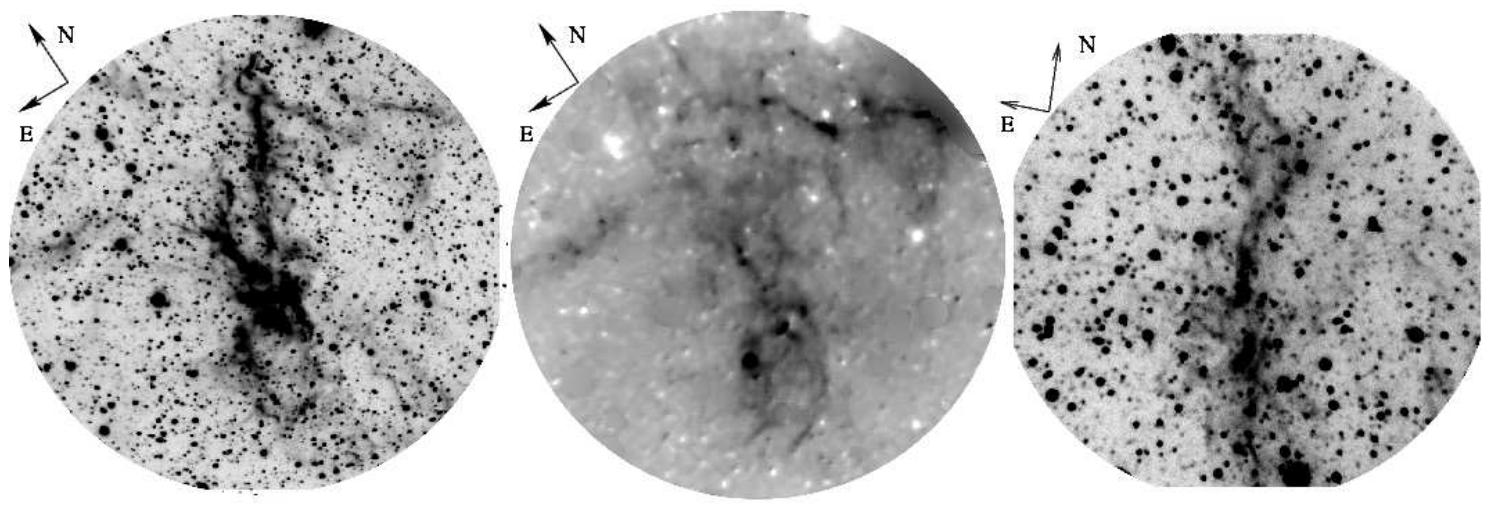

Figure 1: The gas filaments surrounding SS 433. From left to right: the eastern filament $\mathrm{H} \alpha$, [O III] and western filament $\mathrm{H} \alpha$ images. The field size is $5^{\prime}$.

Here we present results of spectroscopy and imaging of the optical gas filaments surrounding SS 433. The eastern and western optical filaments of the radionebula W $50([40,11,6])$ are located in the jet precession cone at a distance of $\approx 50 \mathrm{pc}\left(40^{\prime}\right)$ from SS 433 . The gas filaments are pressed by the jets, they consist of interstellar gas swept out by the jets. The filaments are also exposed directly to the radiation of the supercritical accretion disc at face-on orientation. In the filaments we found strong emission lines (He II, [O III], [Ne III], [Ar III], hydrogen lines) which are formed due to photoionisation and we estimated the face-on luminosity of SS 433 in UV and far UV spectral bands.

In previous spectroscopy of the filaments $([40,22,27,26,6])$ the lines [O III $] \lambda 4959,5007$, [O II] $\lambda 3727,[\mathrm{~N}$ II] $\lambda 6548,6584$ and [S II] $\lambda 6717,6731$ have been detected. The ratios $[\mathrm{N} \mathrm{II}] / \mathrm{H} \alpha=$ $3-5$ and $[\mathrm{S} \mathrm{II}] / \mathrm{H} \alpha=1.5-2.5$ were interpreted $([40,22,27])$ as collisionally excited, probably by shocks. However the observed ratio [O III] $/ \mathrm{H} \beta=5-10$ ([6]) suggests a photoionisation.

Radial velocities of the whole filament system (E+W) were found to be $\mathrm{V}_{h e l}=53 \pm 6 \mathrm{~km} / \mathrm{s}$ in [26] and $\mathrm{V}_{\text {hel }}=56 \pm 2 \mathrm{~km} / \mathrm{s}$ in [6], the both measurements are in very good agreement. However the situation is not such optimistic, for example [26] found that the eastern filaments are receding by $25 \mathrm{~km} / \mathrm{s}$ relative to the western filaments, in apparent disagreement with the orientation found by radio observations. There are probably radial velocity variations in different parts of the filaments. 


\section{Observations and Results}

In the observations we used the SCORPIO focal reducer ([3]) of the Russian 6-m telescope with 2048 x 2048 pixel EEV 42-40 CCD as a detector. We have taken spectra of the eastern and western filaments in August 2006 with spectral range $\lambda \lambda 3700-6500 \AA$ and resolution $11 \AA$. In November 2008 and August 2007 we have taken spectra of the eastern filament in a spectral range $\lambda \lambda 3900-5700 \AA$ and resolution $5 \AA$. A slit width was $1^{\prime \prime}$, a seeing in all observations was $\approx 1.5^{\prime \prime}$. The spectra were reduced using all standard procedures. An accuracy of radial velocity calibration is about $10 \mathrm{~km} / \mathrm{s}$. Imaging of the both filaments has been done with the same device on August 2006 and 2007 using filters $\mathrm{H} \alpha$ (FWHM = $75 \AA$ ), [O III] (220 A) and $\lambda 6063$ (167 ̊) for continuum image.

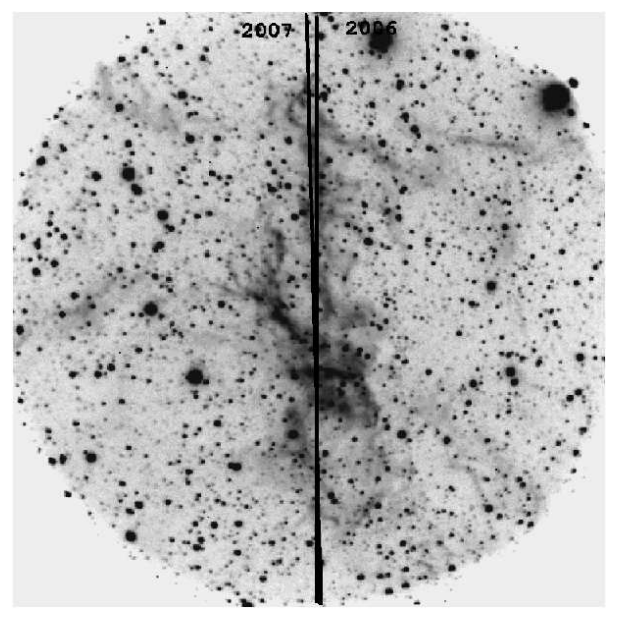

Figure 2: Slit positions in our spectroscopy of the eastern filament.

The eastern and western filaments are shown in Fig. 1. Their distance to SS 433 is about 10 times bigger than the filaments length. Note the clear bow-shock structures in the [O III] image of the eastern filament. The bow-shocks are directed to SS 433 indicating reverse motions of the gas pressed by jets. Fig. 2 shows the slits positions in our spectroscopy made in 2006 and 2007. We chosen these positions very close one another (a few arcseconds only) in order to detect a possible radial velocity difference on a scale of $\approx 0.1 \mathrm{pc}$.

Interstellar extinction to the eastern and western filaments is $\mathrm{A}_{V} \approx 4$ and $\mathrm{A}_{V} \approx 8$ magnitude respectively ([36]), and this is $A_{V} \approx 8$ to SS 433. We confirm these values for the filaments using $\mathrm{H} \alpha / \mathrm{H} \beta$ flux ratios. Our spectrum of the western filament is much more noisy than that of eastern filament because of huge absorption on the western side. Therefore below we study only the eastern filament spectra.

Fig. 3 presents the total spectrum of the eastern filament. The strongest well-known nebular lines [N II] $\lambda 6548,6584$, [S II] $\lambda 6717,6731$, [OI] $\lambda 6300,6360, \mathrm{H} \beta$ and [O III] $\lambda 4959,5007$ are not marked in the fugure. Collisionally excited lines ([N II], [S II], [O I]) are exceptionaly strong in the spectrum. At the same time the high ionization ions ([O III], [Ne III]), [Ar III]) produce very strong lines. The He II $\lambda 4686$ has been discovered for the first time in the W 50 filaments, it is 


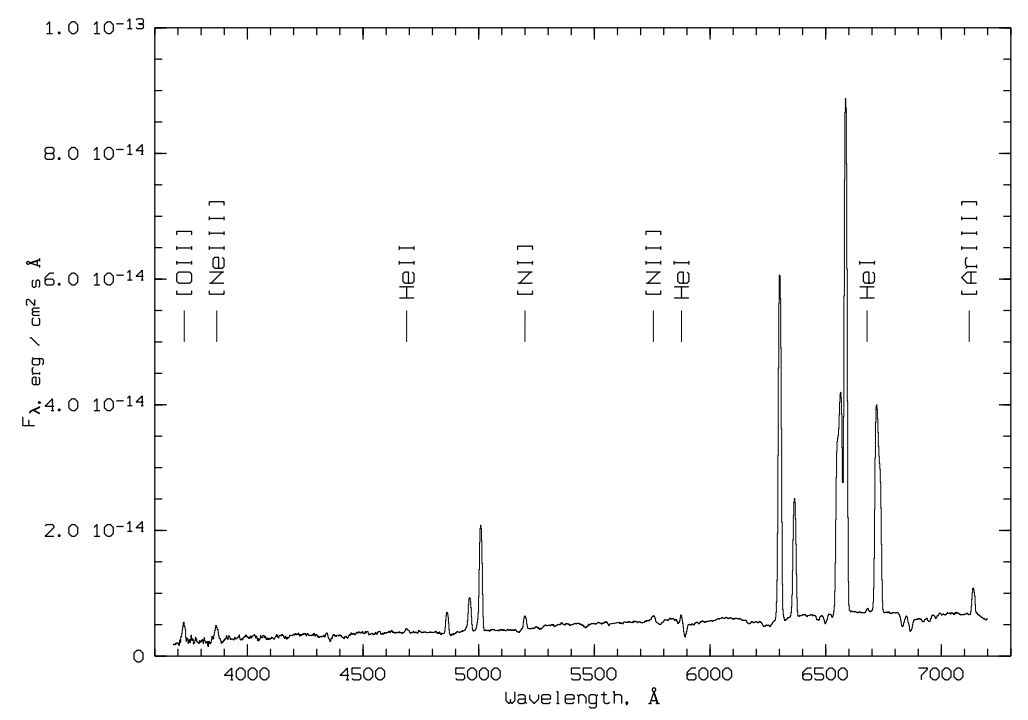

Figure 3: Spectrum of the eastern filament taken with resolution $11 \AA$. Both the absorption features and continum belong to unresolved stars. Obvious stars captured by the slit were excluded at the spectrum extraction.

quite intensive, $\mathrm{He} \mathrm{II} / \mathrm{H} \beta \sim 0.2$. Fig. 4 presents blue spectra of the filament as well as two ULX associated nebulae for comarison. The spectra are quite similar, however the gas excitation and temperature are different (different [SII] $(6716+6731) /(4068+4076)$ and [NII] $(6548+6584) / 5755$ ratios).

Using $\mathrm{H} \alpha / \mathrm{H} \beta$ line ratio in the eastern filanemt we found an interstellar extinction, $\mathrm{A}_{V} \approx 3.8$. $\mathrm{He}$ II and $\mathrm{H} \beta$ line fluxes corrected for the extinction are $\mathrm{F}(\mathrm{HeII})=(4.9 \pm 0.4) \cdot 10^{-17} \mathrm{erg} / \mathrm{cm}^{2} \square$ "s, $\mathrm{F}(\mathrm{H} \beta)=(2.5 \pm 0.07) \cdot 10^{-16} \mathrm{erg} / \mathrm{cm}^{2} \square^{\prime \prime} \mathrm{s}$. After correction for the whole filament surface we estimate the filament line luminosities as L(HeII) $\sim 1.9 \cdot 10^{35} \mathrm{erg} / \mathrm{s}, \mathrm{L}(\mathrm{H} \beta) \sim 8.2 \cdot 10^{35} \mathrm{erg} / \mathrm{s}, \mathrm{L}(\mathrm{O}$ III $) \sim$ $3.8 \cdot 10^{36} \mathrm{erg} / \mathrm{s}$. Electron temperature is estimated to be $\mathrm{T}_{e}=12000-13000 \mathrm{~K}$ from [N II] (6548 $+6584) / 5755$ ratio and $\mathrm{T}_{e}=10500-13500 \mathrm{~K}$ from $[\mathrm{S} \mathrm{II}](6716+6731) /(4068+4076)$ line ratio. If we suggest a shape of the filament as a filament with circular cross-section, we may estimate electron density of the gas, $\mathrm{n}_{e} \sim\left(\mathrm{L}(\mathrm{H} \beta) / \alpha_{r} h v_{H \beta} \mathrm{q} \mathrm{V}\right)^{1 / 2} \sim 70 \mathrm{q}^{-1 / 2} \mathrm{~cm}^{-3}$, where $\mathrm{q}$ is a volume filling factor, $\alpha_{r}$ is a recombination coefficient to produce $\mathrm{H} \beta$ line and $\mathrm{V}$ is a volume. The filament total mass is $\mathrm{M}=\mathrm{m}_{p} \mathrm{~L}(\mathrm{H} \beta) / \alpha_{r} h v_{H \beta} \mathrm{n}_{e} \sim 100 \mathrm{n}_{100}^{-1} \mathrm{M}_{\odot}$, where $\mathrm{n}_{100}$ is electron density in $100 \mathrm{~cm}^{-3}$ units. Note that the mass estimate does not depend directly on the filament volume.

Radial velocity measured along the filament does not show strong variations (Fig. 5) as one may suggest, because the filament dynamically pressed by the jet. The filament length is much greater than its thickness $\sim 1 \mathrm{pc}$ (or even less), this may mean that every piece of the filament interacts with the jet independently. Nevetheless there are not stong velocity variations along the filament. Variations of $\pm 15 \mathrm{~km} / \mathrm{s}$ on a scale comparable with the filament thickness are detected. This may explain some radial velocity disagreements found in the previous spectroscopy. Note that the relative velocities are measured with much better accuracy than absolute velocities. We detect 


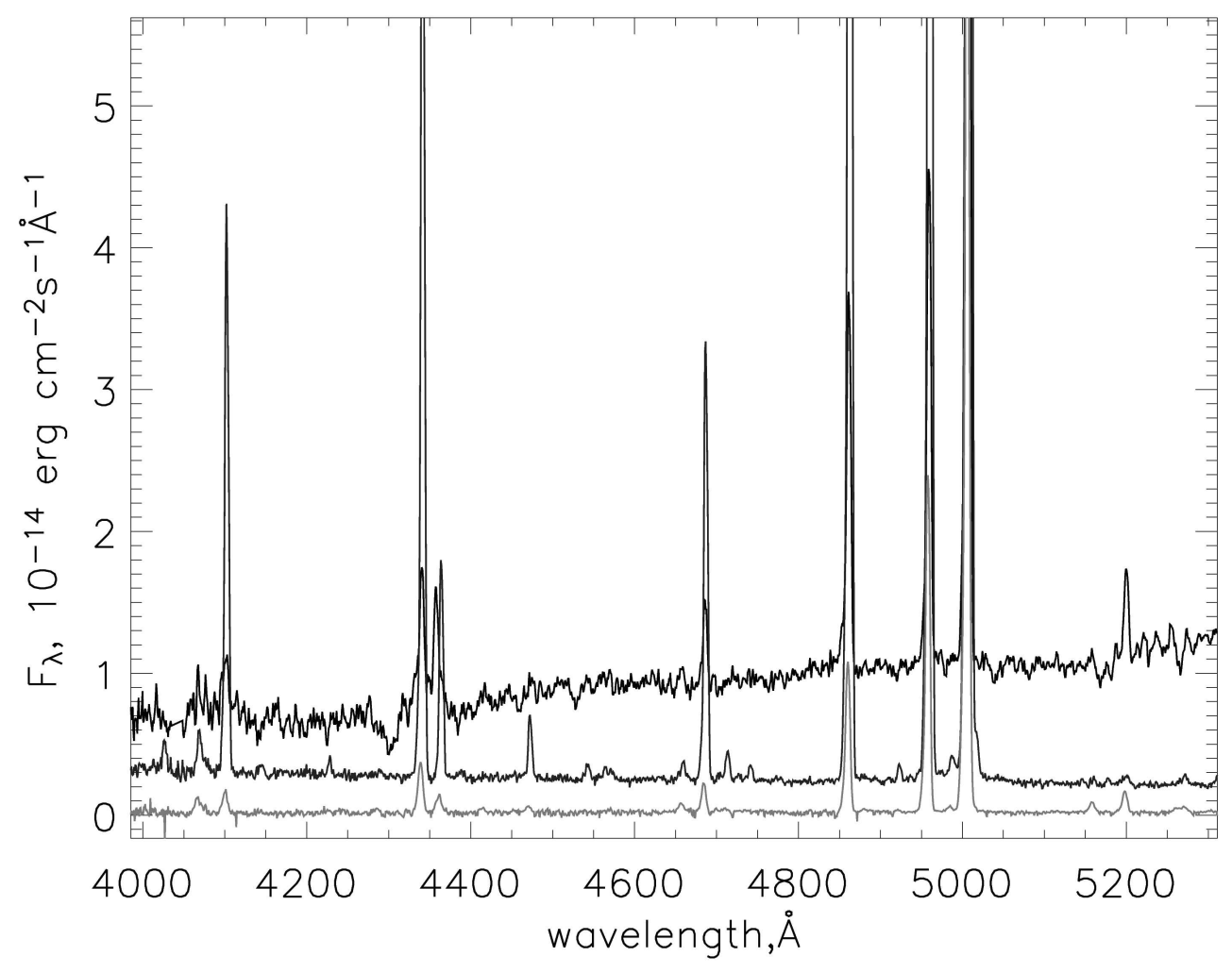

Figure 4: Spectra of the eastern filament (top, multiplied by 3), the nebula associated with the ULX Holmberg II X-1 (middle, multiplied by 25) and the nebula MF 16 of NGC 6946 ULX-1 (bottom, multiplied by 15). The spectra have been taken with the same device.

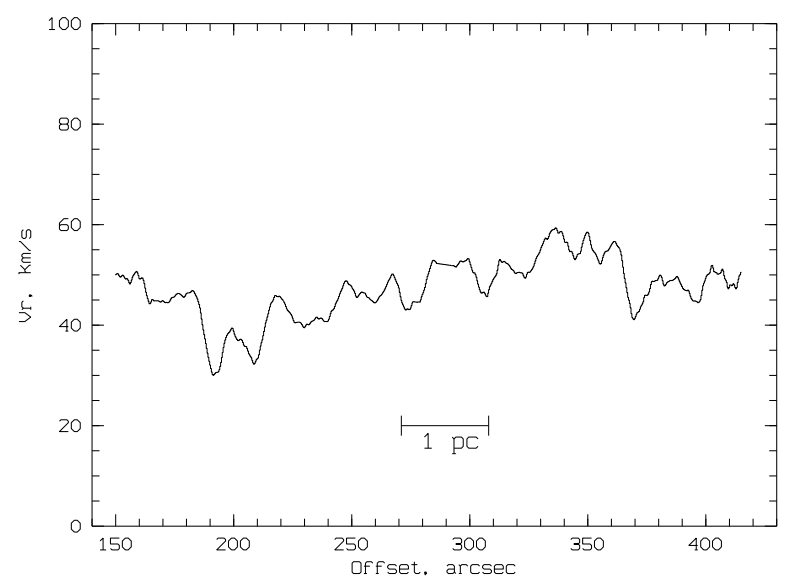

Figure 5: Radial velocity variations along the eastern filament measured in strong [O III] lines. 


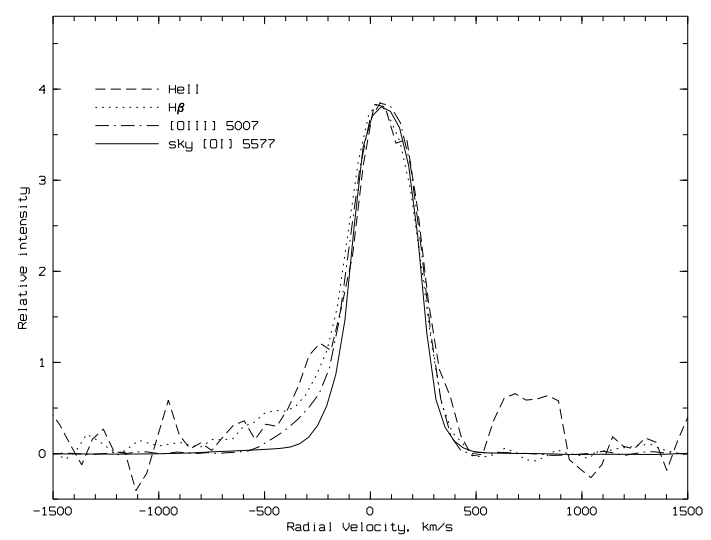

Figure 6: Line profiles of the filaments lines in the total spectrum. The night sky [O I] $\lambda 5577$ line profile is shown for comparison.

a blue wing in emission lines (Fig. 6), thus we confirm the result of [6], where these blue wings were first reported.


Figure 7: Dignostical diagrams for the nebulae associated with ULXs and the eastern filament of W 50. Photoionisation model line ratios are shown for different gas densities and ionization parameters logarithm (from -4 to 0 ) as well as shocks with precursor model line ratios with differents shock velocities in $\mathrm{km} / \mathrm{s}$ and magnetic parameters $(0,1,2,4)$. Details see in $[1,13]$. Dashed lines show shifts to values corrected to the solar abundance.

We compare diagnostical line ratios of the SS 433 filament with those in nebula associated with ULXs using our spectroscopy ([1]). Fig. 7 shows diagnostical diagrams with the model line ratios calculated by [13]. All the nebula positions correspond to the potoionization case with different ionization parameters. Shocks can not produce the line ratios, it is especially clear in the case of He II line, which is very intensive in the nebulae. 

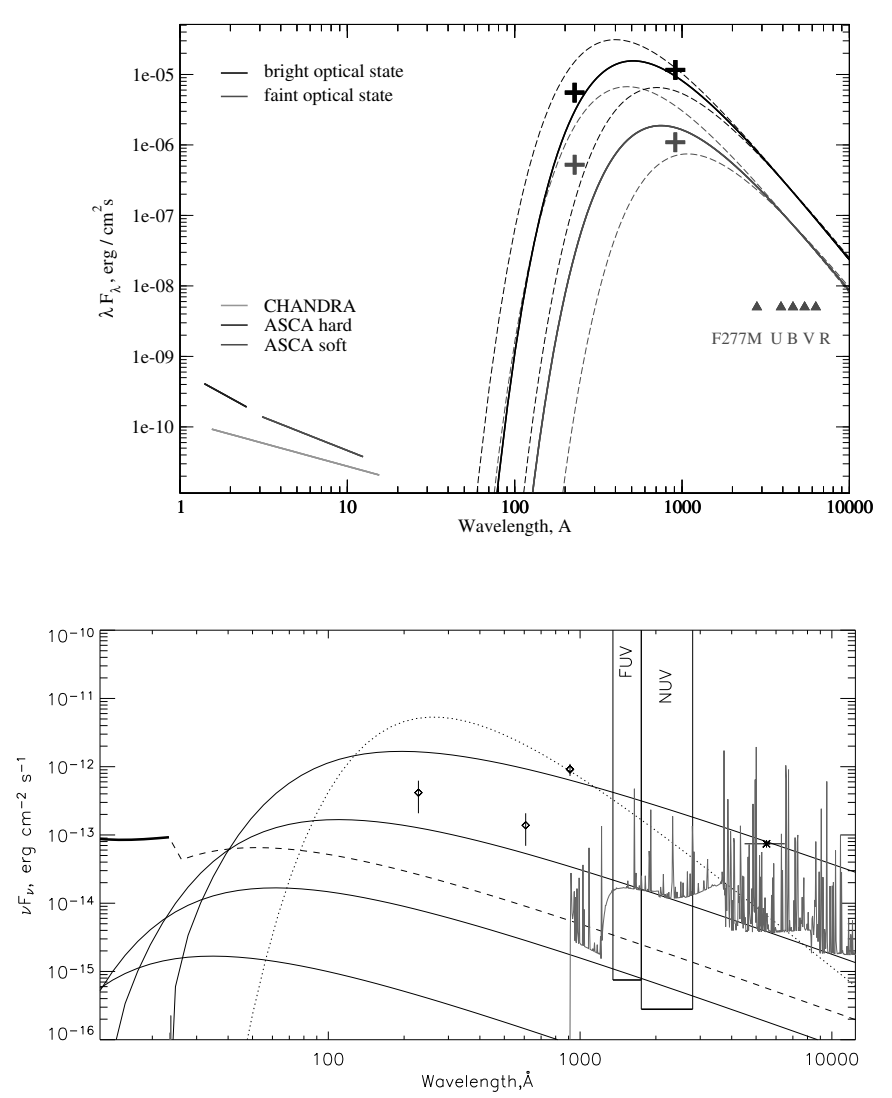

Figure 8: Spectral energy distribution in SS 433 (top) and in NGC6946 ULX-1 (bottom). In SS 433 plot the two solid lines show [10] solutions for bright and faint object states derived from optical and HST photometry, dashed lines indicate possible deviations of these two solutions. Crosses show low limits of ionizing radiation intensity beyond He II $(<228 \AA)$ and $\mathrm{HI}(<912 \AA)$ ionization edges, the upper crosses are estimates of the isotropical source fluxes, the bottom crosses are those for geometrically collimated radiation. X-ray spectral fits obtained with ASCA ([23]) and CHANDRA ([24]) are shown. The NGC6946 ULX-1 plot taken from [2]. Similar estimates of ionizing fluxes beyond the He II, He I and H I ionization edges are shown by diamonds with error bars. X-ray spectral fit by [35] (power law (solid line) plus MCD component (dashed line)) is shown. The best CLOUDY model fit to observed nebula spectrum is shown by dotted line as a black body $\left(\mathrm{T}=1.4 \cdot 10^{5} \mathrm{~K}\right)$. Four MCD models are shown (solid lines) for the black hole masses $10,100,1000$ and $10^{4} M_{\odot}$ (see [2] for more detals). GALEX photometric limits (FUV and NUV bands) are shown for $\mathrm{S} / \mathrm{N}=5$ and an exposure time $10^{5} \mathrm{sec}$.

Using the He II and $\mathrm{H} \beta$ luminosities of the filament we estimate corresponding ionizing luminosities of the supercritical accretion disc in SS 433. In the case of isotropical radiation of SS 433 disk we obtain the estimates $\mathrm{L}(<228 \AA) \geq \mathrm{L}(\mathrm{He}$ II $)\left(\mathrm{h} v_{228} / \mathrm{h} v_{4686}\right)\left(\alpha_{t o t} / \alpha_{4686}\right) \sim 1.2 \cdot 10^{40} \mathrm{erg} / \mathrm{s}$ and $\mathrm{L}(<912 \AA) \geq \mathrm{L}(\mathrm{H} \beta)\left(\mathrm{h} v_{912} / \mathrm{h} v_{H \beta}\right)\left(\alpha_{t o t} / \alpha_{H \beta}\right) \sim 2.8 \cdot 10^{40} \mathrm{erg} / \mathrm{s}$, where we used a total and the line recombination coefficients for the case $\mathrm{B}$ of gas nebulae. Actually the UV luminosity estimates are low limits because of possible not total absorption of the ionizing radiation in the 
filament. In a case of non-izotropical UV radiation of the supercritical accretion disc, we have to introduce a factor $\Omega_{f} / 2 \pi$ in the luminosity estimates. With $\theta_{f}=50^{\circ}$ we obtain estimates of the ionizing luminosities as $\mathrm{L}(<228 \AA) \sim 1.1 \cdot 10^{39} \mathrm{erg} / \mathrm{s}$ and $\mathrm{L}(<912 \AA) \sim 2.6 \cdot 10^{39} \mathrm{erg} / \mathrm{s}$.

In Fig. 8 we show spectral energy distribution of SS 433, where the UV flux estimates put together with [10] black body fits, with $\mathrm{T}=72000 \pm 20000 \mathrm{~K}$ for the bright state and $\mathrm{T}=49000 \pm$ $20000 \mathrm{~K}$ for the faint state of SS 433. Positions of the spectral bands used by [10] are shown in the figure. Note that these black bodies as well as the observed X-ray spectrum were derived for observed nearly edge-on orientation of SS 433, whereas the UV estimates are for the face-on orientation, The filament sees the disc face-on. In the figure we also present the same SED estimates for NGC6947 ULX-1 ([2]), where the UV fluxes were derived from the nebula MF 16 associated with the ULX. The both objects need strong UV radiation to produce observed emission lines in the nebula. The observed X-ray spectrum of NGC6946 ULX-1 is about the same intensive as predicted UV spectrum indicating the probable flat spectrum of the ULX (what is actually expected to be $([34,33]))$. However the observed X-ray luminosity of SS 433 is $\sim 5$ orders of magnitude fainter than its real UV luminosity.

We conclude that SS 433 has to be ultraluminous in UV and far UV bands with the liminosity $\mathrm{L}_{U V} \sim 10^{40} \mathrm{erg} / \mathrm{s}$, the source temperature is $\mathrm{T} \sim 10^{5} \mathrm{~K}$. About the same spectrum has been found from observed (HST/HSP and optical bands) in SS 433 ([10]) at its nearly edge-on orientation. We confirm thus the previous luminosity and temperature estimates, SS 433 is really extremely luminous. This means also that the supercritical accretion disc UV radiation in SS 433 is roughly isotropical and its spectrum can be represented by a black body radiation. All these findings confirm the original Shakura - Sunyaev's idea [37] on the supercritical accretion discs which produce strong winds.

\section{References}

[1] Abolmasov P., Fabrika, S., Sholukhova, O., Afanasiev, V. L., 2007, Astronomical Bull., 62, 36

[2] Abolmasov P., Fabrika, S., Sholukhova, O., Kotani, T., 2008, Publ. Astron. Soc. Jap., in press.; astro-ph/0809.0409

[3] Afanasiev V. L., Moiseev A. V., 2005, Astron. Lett, 31, 194

[4] Blundell, K. M. \& Bowler, M. G., 2004. Astrophys. J., 616, L15

[5] Blundell, K. M., Bowler, M. G., Schmidtobreick, L., 2008, Astrophys. J., 678, L47

[6] Boumis, P., Meaburn, J., Alikakos, J. et al., 2007, Mon. Not. Roy. Astron. Soc., 381, 308

[7] Calzetti D., Kinney A. L., Ford H. et al., Astron. J., 110, 2739

[8] Cherepashchuk, A. M., Aslanov, A. A., Kornilov, V. G., 1982, Sov. Astron., 26, 697

[9] Cherepashchuk, A. M., Sunyaev, R. A., Fabrika, S. N. et al., 2005, Astron. Astrophys., 437, 561

[10] Dolan, J. F., Boyd, P. T., Fabrika, S. et al., 1997, Astron. Astrophys., 327, 648

[11] Dubner G. M., Holdaway M., Goss W. M., Mirabel I. F., 1998 Astron. J. 116, 1842

[12] Eggum, G. E., Coroniti, F. V., Katz, J. I., 1988, Astrophys. J., 330, 142

[13] Evans, I., Koratkar, A., Allen, M., et al., 1999, Astrophys. J., 521, 531 
[14] Fabrika S. 2004, Astrophys. and Space Phys. Rev., 12, 1

[15] Fabrika S., Sholukhova O. 1995, Astrophys. and Space Sci., 226, 229

[16] Fabrika S., Mescheryakov A. 2000, JENAM-2000, 9th European and 5th Euro-Asian Astronomical Society Conference, Moscow, May 29 - June 3, 2000, Meeting Abstract, p. 74.

[17] Fabrika S. \& Mescheryakov A., 2001, in Galaxies and their Constituents at the Highest Angular Resolution (Ed. R.T. Schilizzi), IAU Symp. N 205 (Manchester, United Kingdom), p. 268

[18] Fabrika, S., Karpov, S., Abolmasov, P., Sholukhova, O., 2006, in "Populations of High Energy Sources in Galaxies", IAU Symp. N 230, E. J. A. Meurs, G. Fabbiano (eds.), 278

[19] Gies, D. R., Huang, W., McSwain, M. V, 2002, Astrophys. J., 578, L67

[20] Hillwig, T. C. \& Gies, D. R., 2008, Astrophys. J., 676, L37

[21] Katz, J. I., 1987, Astrophys. J., 317, 264

[22] Kirshner, R. P. \& Chevalier, R. A., 1980, Astrophys. J., 242, L77

[23] Kotani, T., Kawai, N., Matsuoka, M., Brinkmann, W., 1996, Publ. Astr. Soc. Jap., 48, 619

[24] Marshall, H. L., Canizares, C. R., Schulz, N. S., 2002, Astrophys. J., 564, 941

[25] Massey P., McNeill R. T., Olsen K. A. G., et al., 2007, Astron. J., 134, 2474

[26] Mazeh, T. Aguilar, L. A., Treffers, R. R. et al., 1983, Astrophys. J., 265, 235

[27] Murdin, P., Clark, D. H., 1980, Mon. Not. Roy. Astron. Soc., 190, 65P

[28] Murdin, P., Clark, D. H., Martin, P. G., 1980, Mon. Not. Roy. Astron. Soc., 193, 135

[29] Mushotzky, R., 2006, Adv. Space Res., 38, 2793

[30] Neese, C. L., Armandroff, T. E., \& Massey, P., 1991, in "Wolf-Rayet Stars and Interrelations with Other Massive Stars in Galaxies", Edited by Karel A. van der Hucht and Bambang Hidayat, IAU Symp. N 143, p. 651

[31] Ohsuga, K., Mori, M., Nakamoto, T., Mineshige, S., 2005. Astrophys. J., 628, 368

[32] Okuda, T., Teresi, V., Toscano, E., Molteni, D., 2005, Mon. Not. Roy. Astron. Soc., 357, 295

[33] Poutanen, J., Lipunova, G., Fabrika, S., et al., 2007, Mon. Not. Roy. Astron. Soc., 377, 1187

[34] Roberts, T. P., 2007, Astrophys. and Space Sci., 311, 203

[35] Roberts, T. P, Colbert, E. J. M. 2003, Mon. Not. Roy. Astron. Soc., 341, 49

[36] Schlegel, D. J., Finkbeiner, D. P., Davis, M., 1998, Astrophys. J., 500, 525

[37] Shakura N. I. \& Sunyaev R. A., 1973, Astron. Astrophys., 24, 337

[38] Swartz, D. A., Ghosh, K. K., Tennant, A. F., Wu, K., 2004, Astrophys. J. Suppl. Ser., 154, 519

[39] Wagner, R. M., 1986, Astrophys. J., 308, 152

[40] Zealey, W. J., Dopita, M. A., Malin, D. F., 1980, Mon. Not. Roy. Astron. Soc., 192, 731 